\title{
Targeting care in Barrett's oesophagus
}

\author{
Authors: Sebastian Zeki ${ }^{A}$ and Rebecca C Fitzgerald ${ }^{B}$
}

Barretts oesophagus represents the most significant risk factor for the development of oesophageal adenocarcinoma (OAC), although the majority of patients will not develop cancer. However, early detection of OAC and its precursors significantly improves outcome and underlines the importance of endoscopic surveillance programmes. Clearly there is a discrepancy between the small number of people who need to undergo surveillance because they are at significant progression risk, and the large number that do. Research is therefore now concentrated on risk stratification. Currently such stratification is currently based on clinical findings, endoscopic diagnosis and histopathological grade. Histopathology can be imperfect and is likely to require molecular confirmation of different grades, thus molecular stratification is becoming more important in this regard and p53 immunohistochemistry is already clinically useful, with other molecular biomarkers likely to prove beneficial in the future. The hope is that non-endoscopic methods, such as the Cytosponge ${ }^{\mathrm{TM}}$ may be able to combine molecular biomarkers with histopathology and therefore perhaps benefit a population screening as well as a surveillance programme.

KEYWORDS: Barrett's oesophagus, oesophageal adenocarcinoma, molecular stratification

\section{Introduction}

Oesophageal adenocarcinoma (OAC) has a high mortality with a median overall survival of 1 year; even when a curative modality is attempted, for cases without metastases to distant organs, 5 -year survival remains poor. ${ }^{1}$ There has been a dramatic increase in this disease in the western world over the past 3 decades and, although more recent data have demonstrated that the incidence of OAC might now be plateauing worldwide, in the UK the incidence is increasing and outcome remains poor. $^{2}$

One of the most commonly associated risk factors with OAC is the development of Barrett's oesophagus (BO). BO is thought to be the result of a metaplasia from the normal squamous lining of the oesophagus to a columnar, glandular

Authors: A postdoctoral research fellow, University of Cambridge, Cambridge, UK; ${ }^{B}$ professor of cancer prevention, University of Cambridge, Cambridge, UK phenotype. Although the cellular origin of $\mathrm{BO}$ has not yet been clarified, there is strong evidence from epidemiological and experimental data that the aetiology is related to refluxate. BO is characterised by several cell lineages, including intestinal metaplasia, which is the hallmark and the type that predisposes most to adenocarcinoma. The development of cancer is generally gradual through a metaplasia-dysplasiacancer sequence, which is a well-established progression sequence for several different, particularly epithelial, cancers. The sequence is often considered to be temporally linear: metaplasia develops into dysplasia, either of low or high grade (LGD or HGD, respectively), which is the precursor lesion to adenocarcinoma. Importantly, if adenocarcinoma is detected when it is confined to the mucosa (so-called intramucosal carcinoma'), the 5 -year survival rate is $95 \%$, whereas invasion into the deeper submucosa increases the risk of lymph node metastases considerably (from 5\% to $40 \%$ depending on degree of submucosal invasion) and increases the 5-year mortality. ${ }^{3,4}$ This is the premise for the importance of early detection in this disease.

\section{Cancers take years to evolve - understanding precursor lesions and early cancers as targets}

Sporadic colon cancer, for example, is known to take 5-15 years to develop and is preceded by adenomas comprising LGD or HGD. ${ }^{5}$ Prostate, cervical, bladder and breast cancers are all epithelial cancers with precursor lesions that take many years to evolve before invasive cancer occurs (Table 1).

Therefore, detectable precursor lesions represent an ideal target for the prevention of cancer development. Such strategies are already integral to several cancer prevention programmes. For example, the UK Bowel Cancer Surveillance Programme screens for the detection of cancer and uses a guideline-based follow-up of adenoma detection and eradication. ${ }^{6}$ Another example is the Japanese model of gastric cancer screening. Since the introduction of the national gastric cancer screening programme in Japan, the detection of early gastric cancer has increased significantly with a consistent decrease in patient mortality. ${ }^{7}$

Given the importance of early cancer detection and the possibility of treating cancer precursor lesions to prevent cancer development, the question arises concerning how we can best achieve this goal given the low progression rate in $\mathrm{BO}$. At the two ends of the spectrum should we be investigating only patients with upper gastrointestinal (GI) symptoms, those who are considered to be high risk based on clinicopathological features, or should we consider population screening? 


\begin{tabular}{lll}
\multicolumn{2}{l}{ Table 1. Examples of cancers and their associated } \\
precursor lesions.
\end{tabular}

\section{The evidence for surveillance}

Several studies have demonstrated that the overall risk of developing OAC in patients with non-dysplastic BO is small, with two large population-based cohort studies concluding that the per patient risk of OAC is between $0.12 \%$ and $0.16 \%$ per year. Patients were at greater risk if goblet cells were present (intestinal metaplasia) and with longer Barrett's segments $(>3 \mathrm{~cm}){ }^{8,9}$

Given the low rate of OAC development and that surveillance programmes exist in most western countries, it is worth considering what the impact of surveillance regimes has been on the development of OAC or its mortality. Although several studies found a positive impact on survival, ${ }^{10,11}$ the most recent study from Kaiser Permanente in the USA argues against the need for surveillance. ${ }^{12}$ This case-control study by Corley et al matched 38 OAC deaths by age, year and place of diagnosis, sex and race, with 101 controls (patients with Barrett's but who did not die from oesophageal adenocarcinoma by the end of the study, although patients in this group could still have oesophageal cancer) from a large database kept between 1995 and 2007. The authors concluded that a surveillance gastroscopy within 3 years of the diagnosis of cancer did not decrease the risk of death from OAC. This study highlights some of the challenges related to determining surveillance impact in an area where endoscopic technologies and treatments change and where surveillance might not be standardised. Of the patients in this study who developed cancer, $47.4 \%$ had previously been found to have dysplasia before cancer development and, in those who had developed cancer, approximately 50\% had cancer diagnosed at an advanced stage, indicating that the quality of the surveillance was poor. Therefore, their study underlines the need for high-quality surveillance according to strict protocol. Furthermore, whereas treatment by oesophagectomy would
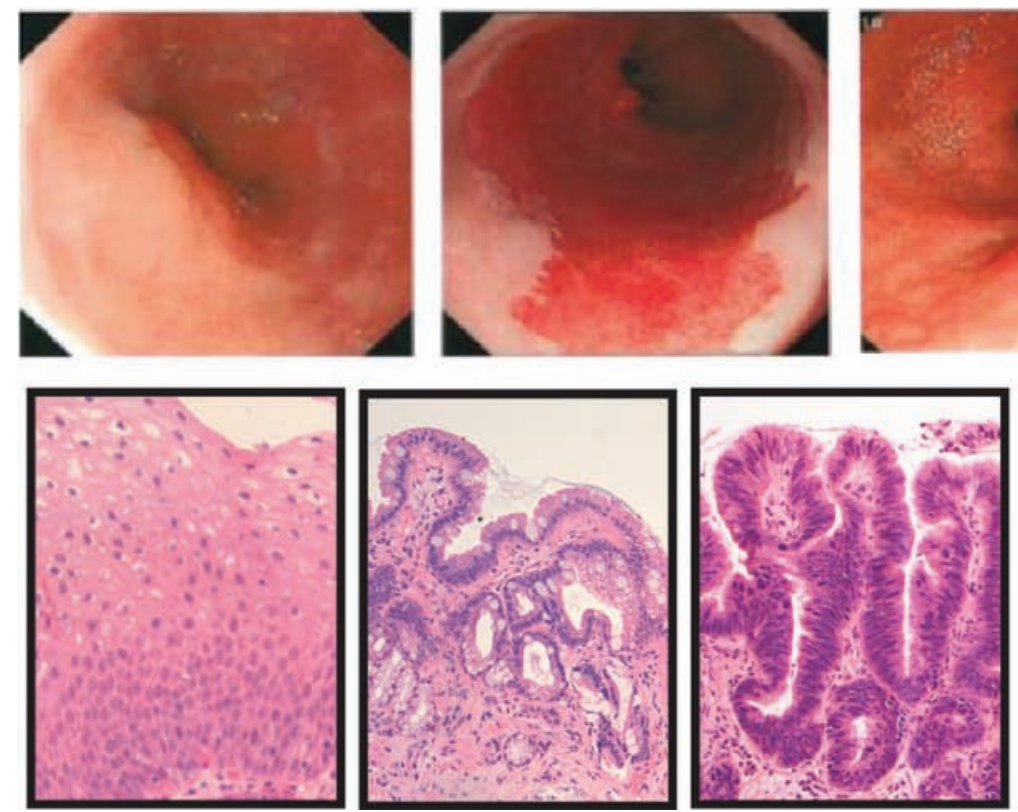

Non-dysplastic Barrett's oesophgus

Squamous

Low grade dysplasia
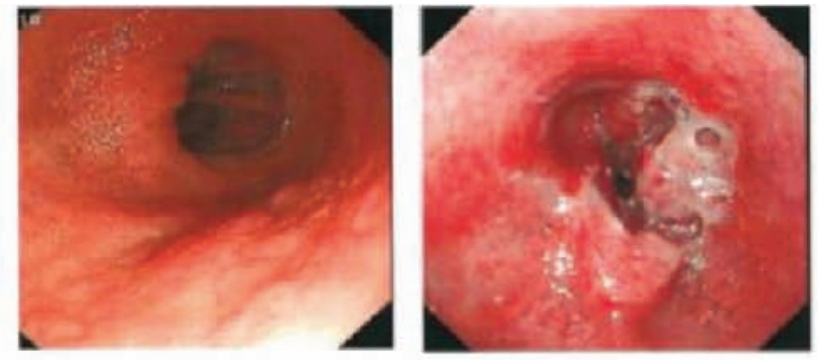

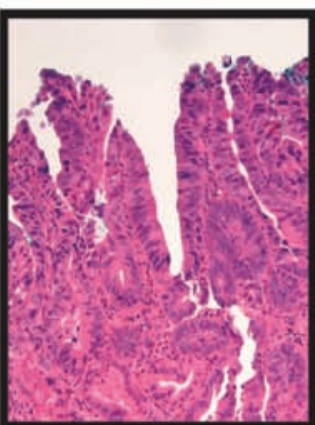

High grade dysplasia

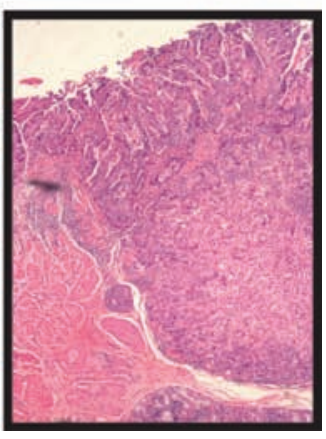

Adenocarcinoma

Fig 1. An endoscopic (top panels) and histological (bottom panels) description of the development of oesophageal adenocarcinoma in patients with Barrett's oesophagus. The initial lining of the oesophagus comprises squamous cells. These are then replaced by non-dysplastic columnar cells with or without goblet cells. In a few patients, further molecular and genetic aberrations result in low- and then high-grade dysplasia. The latter is the immediate precursor of, and often coexists with, adenocarcinoma. 


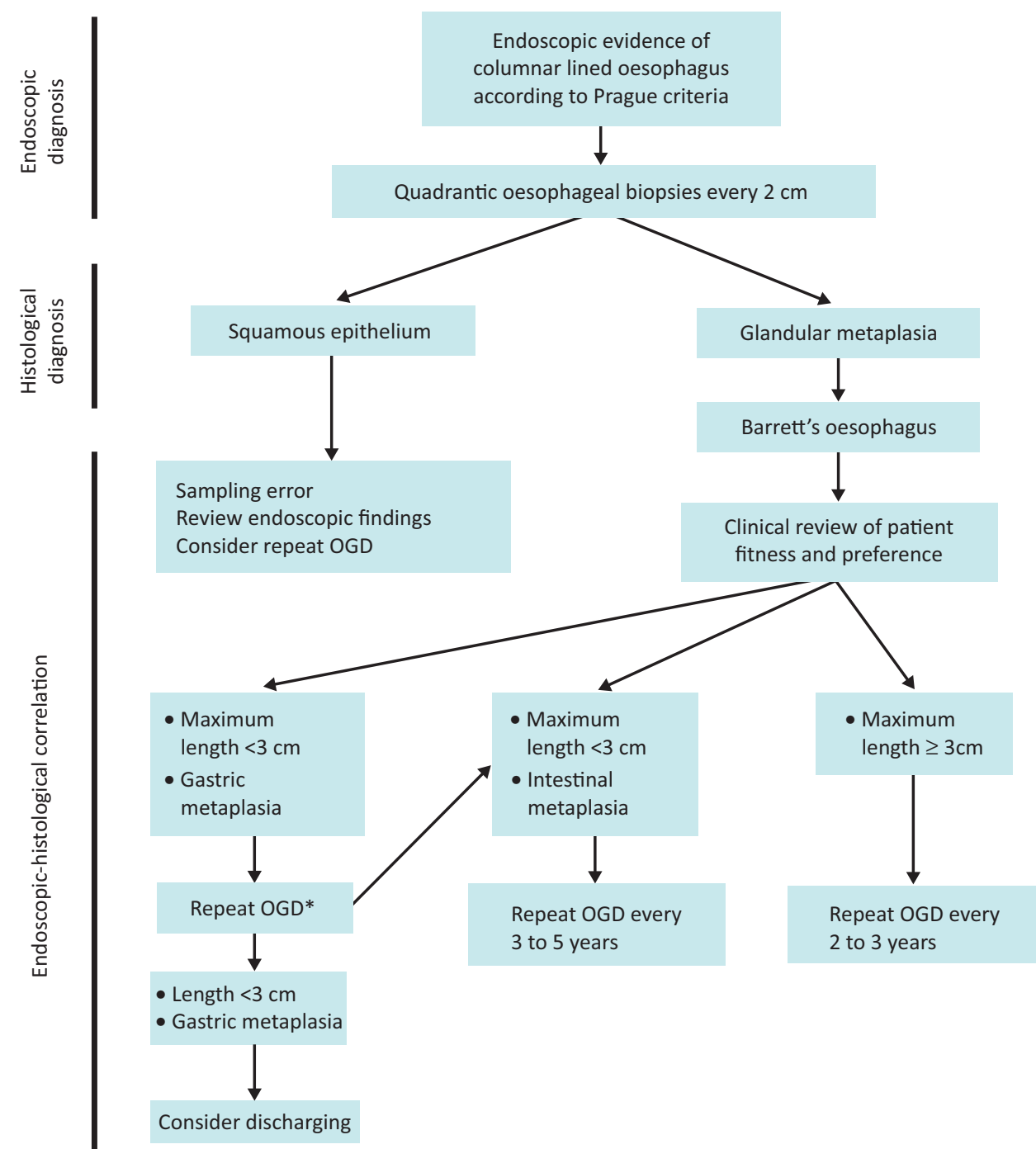

Fig 2. Algorithm for the endoscopic evaluation and follow-up for patients with non-dysplastic Barrett's oesophagus. *Interval depends on the degree of clinical confidence about diagnosis (accuracy of endoscopic report and number of biopsies). Reproduced with permission from Gut. ${ }^{13} \mathrm{OGD}=$ oesophago-gastro-duodenoscopy. have been available for HGD in this historical cohort, the more successful endoscopic therapies, such as radiofrequency ablation, which are now preferred for early disease, would have only been available after this cohort was collected. Thus, this interesting study not only highlights the difficulty of assessing historical data and retrospective studies, but also underlines the importance of stratifying patients according to their risk of developing cancer, especially given the ability of current endoscopic therapy to eradicate earlier oesophageal precursor lesions.

\section{The growing emphasis on targeting the right patients} for surveillance

Therefore, the standardisation of clinical care for $\mathrm{BO}$ is an important part of assessing the true impact of surveillance programmes. The UK guidelines for the diagnosis and treatment of Barrett's have recently been updated. ${ }^{13}$ Important evidence-based changes have been introduced that start to clarify how patients can be risk stratified according to clinicopathological features (Fig 2).

\section{Clinical stratification}

The Prague classification for endoscopic reporting of Barrett's segments, which reports the circumferential extent (denoted $\mathrm{C}$ for circumferential and the length in $\mathrm{cm}$ ) as well as total extent (taking tongues of Barrett's into account $(\mathrm{M})$ ) from the gastrooesophageal junction, is now a standard endoscopic reporting descriptor. This should be combined with the Paris classification, which standardises the description of visible oesophageal lesions. As regards risk stratification, several studies have demonstrated that patients with longer Barrett's sections $(>3 \mathrm{~cm})$ at endoscopy are considered to be at higher risk $^{14-16}$ of OAC progression and, therefore, the intensity of endoscopic surveillance is modified between 2 and 5 years accordingly.

\section{The need for high-quality, high-resolution endoscopy}

The need for accurate and high-quality endoscopy has also increased in importance as treatment for early OAC becomes more accurate and targeted. Much progress has been made 
High-resolution endoscopy (HRE)

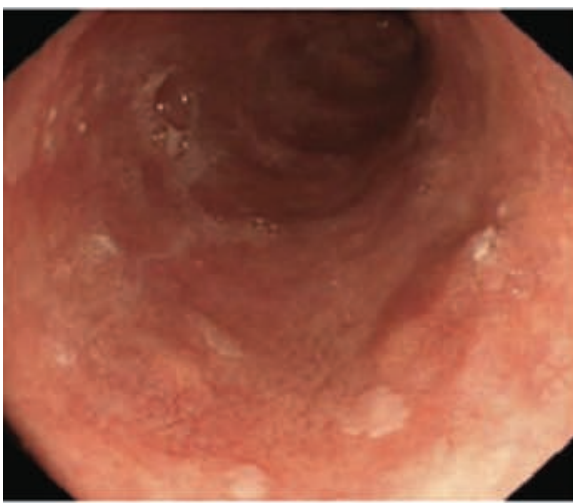

Autofluorescence imaging (AFI)

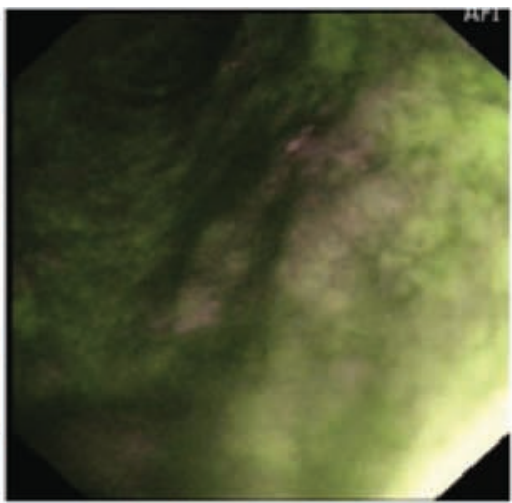

Narrowband imaging-zoom (NBI-Z)

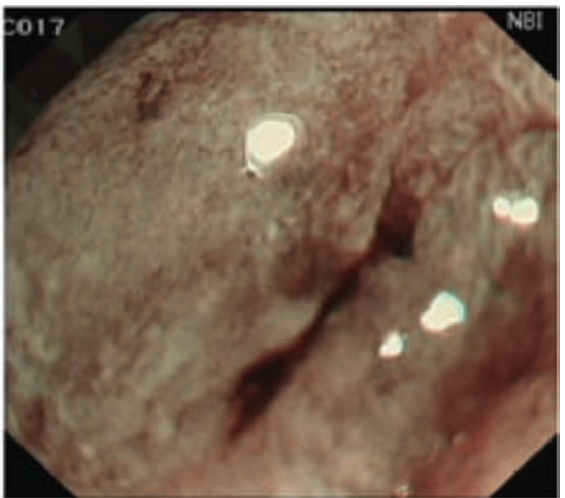

Fig 3. Different endoscopic modalities for the examination of a Barrett's segment. AFI = autofluorescence imaging; HRE = high-resolution endoscopy; NBI-Z = narrowband imaging-zoom.

with endoscopic modalities for early OAC and HGD treatment, but these treatments rely on the recognition of lesions, which can be subtle. The endoscopist's tool-kit contains several modalities to enhance lesion recognition, including autofluorescence, narrowband imaging, confocal microscopy and chromoendoscopy (Fig 3). Although still experimental, the ability to target suspicious areas at endoscopy reduces unnecessary sampling and can increase the yield of potentially treatable areas. ${ }^{17}$ Given that modalities such as radiofrequency ablation with or without endoscopic or submucosal mucosal resection are now curative in most cases, including early OAC, such targeting is of paramount importance.

\section{Histological stratification}

Histologically, although the presence of intestinal metaplasia is not a prerequisite for the diagnosis of $\mathrm{BO}$, as it is in North America, its presence might also indicate a higher risk of OAC development ${ }^{9,18,19}$ and, therefore, endoscopic follow-up of these patients is also stratified accordingly. A key emphasis is placed on the fact that, if the patient has gastric metaplasia without goblet cells, and the Barrett's segment is less than 3 $\mathrm{cm}$, then discharge from surveillance should be considered because the risk of endoscopy might outweigh the benefit of surveillance. Given the importance of a histological diagnosis, the guidelines also stress the need for histopathological quality control, particularly in relation to dysplasia grade, because this can trigger a therapeutic intervention with endoscopic therapy. The detection of LGD carries a hazard ratio of 5.67 for the development of HGD and OAC combined. ${ }^{9}$ Historically there have also been different treatment pathways based on the detection of LGD and HGD; thus, the lack of consensus in diagnosing dysplasia subtypes between histopathologists has been of concern. Patients with HGD will all need to be discussed at the local multidisciplinary team meeting after consensus review and, depending on endoscopic and histopathological features, might undergo radiofrequency ablation, endoscopic mucosal resection, surgery or a combination thereof. However, patients with LGD were advised to undergo a gastroscopy every 6 months until two consecutive endoscopies demonstrated non-dysplastic
Barrett's or the patient developed HGD. Several studies have demonstrated kappa values for interobserver agreement in the diagnosis of LGD as low as $0.18 .{ }^{20}$ In fact, although LGD without consensus is reported to have an incidence rate of later OAC development of $0.49 \%$, at least one study has demonstrated that, with consensus between at least two histopathologists, the incidence rate increases to $13.4 \% .^{21}$ A recent randomised controlled trial has demonstrated that with consensus between expert GI pathologists, patients who undergo ablation for LGD are significantly less likely to develop adenocarcinoma and therefore the ablation of Barrett's containing confirmed LGD is now recommended. ${ }^{22}$

\section{Can molecular biology help histopathological diagnosis? Molecular stratification}

Based on the above discussion, strategies to increase the consensus around a diagnosis of LGD would be helpful. The protein product, p53 of the tumour suppressor gene TP53, which is often mutated in the progression to OAC, is now validated as an adjunct to help clarify the diagnosis of LGD. An abnormal pattern is associated with an increased risk of OAC progression in patients with BO (Fig 4). It is also a better predictor of neoplastic progression compared with the histological diagnosis of LGD and has the benefit of good interobserver agreement. ${ }^{23,24}$

\section{The future - considering population screening \\ Cytosponge $^{\mathrm{TM}}$ for population screening}

Most of the work in $\mathrm{BO}$ has been undertaken in patients who have already been found to have the condition and, usually, they have presented with upper GI symptoms of reflux. However, given that the population prevalence of $\mathrm{BO}$ is estimated to be around $2 \%$ of the total population in the western world, over $80 \%$ of patients with $\mathrm{BO}$ will remain undiagnosed. ${ }^{25,26}$ To reduce the incidence of $\mathrm{OAC}$, it follows that we should also concentrate on diagnosing the population at risk through screening as well as risk stratifying those with diagnosed BO.

Endoscopic population screening would be unfeasible and, therefore, recent trials have assessed novel technologies for 
Fig 4. Immunohistochemistry for p53. (a) Overexpression of p53. (b) Absent $\mathrm{p} 53$ pattern. Both are pathological.
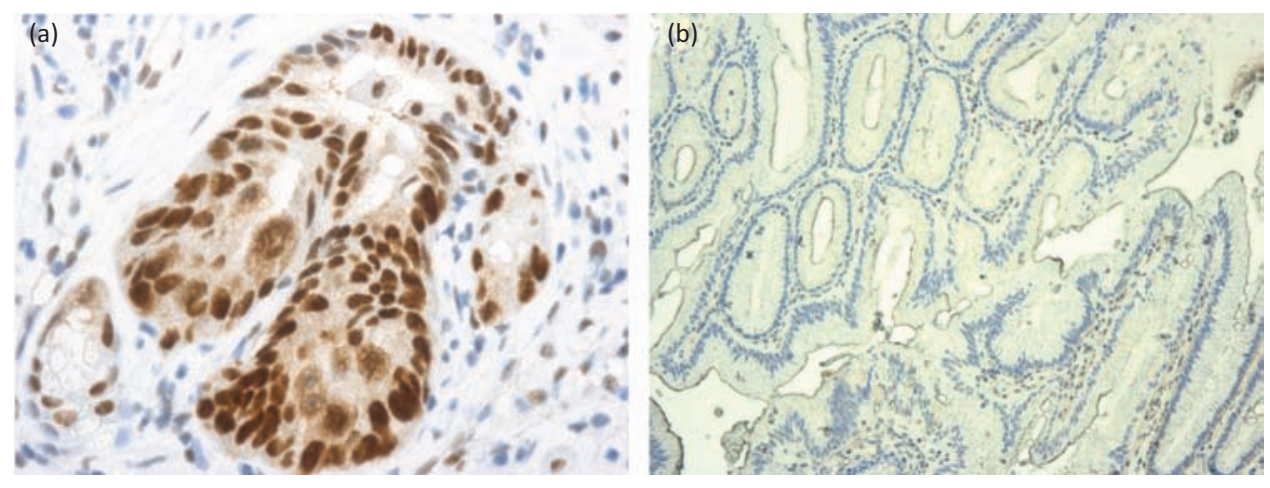

this purpose, including ultrathin transnasal endoscopy and non-endoscopic technologies, such as the Cytosponge ${ }^{\mathrm{TM}}$. The Cytosponge ${ }^{\mathrm{TM}}$ is a capsule on a string, which the patient swallows. Once in the stomach, the capsule dissolves to release a spherical sponge mesh that can then be retrieved using the string; during its retrieval, approximately 500,000 oesophageal cells accumulate within the mesh. The administration and retrieval of the Cytopsonge ${ }^{\mathrm{TM}}$ takes 5 minutes and can be performed in the GP surgery. These cells can then be prepared for histological and molecular analyses with immunohistochemical analysis of a Barrett's marker, trifoil factor 3 (TFF3), proving crucial for the success of this test.

The Cytosponge ${ }^{\mathrm{TM}}$ test combined with TFF3 detection was compared with endoscopy and biopsy in a primary care cohort study of just over 500 patients. ${ }^{27}$ The sensitivity and specificity of Cytosponge ${ }^{\mathrm{TM}}$ and TFF3 detection for the detection of BO was $73.3 \%$ and $93.8 \%$, respectively for short-segment BO $(<1$ $\mathrm{cm}$ circumferential length) and increased to $90 \%$ and $93.5 \%$, respectively for longer segments $>3 \mathrm{~cm}$. This compares well with tests used in other national cancer screening programmes. A second study of over 1,000 patients is now being completed to evaluate this approach further and the results are awaited (in a

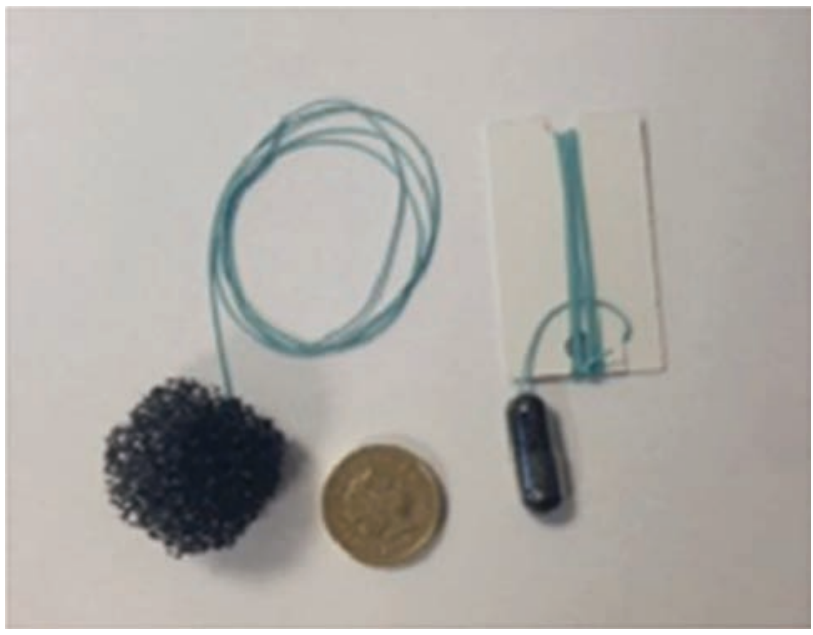

Fig 5. The Cytosponge ${ }^{\mathrm{T}}$. The Cytosponge $\mathrm{e}^{\mathrm{TM}}$ is swallowed as a capsule. The soft mesh expands once in the stomach and, on withdrawal, the mesh traps a sample of oesophageal cells, which can then be analysed.
Cancer Research UK-funded and National Institute of Health Research portfolio study called 'BEST2').

The Cytosponge ${ }^{\mathrm{TM}}$ raises the possibility of population screening for BO while uncoupling endoscopy units from the burden of carrying out what would necessarily be a large screening programme. An obvious next step is to ask whether the Cytosponge ${ }^{\mathrm{TM}}$ test could be taken further so that, once the $\mathrm{BO}$ has been detected, further analyses might enable patients with $\mathrm{BO}$ to be stratified as high or low risk for progression to OAC. This possibility will be evaluated in the recently completed BEST2 study.

Using non-endoscopic means, such as the Cytosponge ${ }^{\mathrm{TM}}$, to both diagnose and risk stratify those with BO might not only reduce the burden on patients having to undergo endoscopic surveillance for $\mathrm{BO}$, but also reduce the healthcare cost burden by reducing the overall number of endoscopies needed. ${ }^{28}$ A future paradigm is demonstrated in Fig 6, whereby symptomatic patients with heartburn would undergo Cytosponge $^{\mathrm{TM}}$ assessment and, if found to contain Barrett's

Heartburn sufferers:
$10 \%$ of adults
aged $50-70$ years
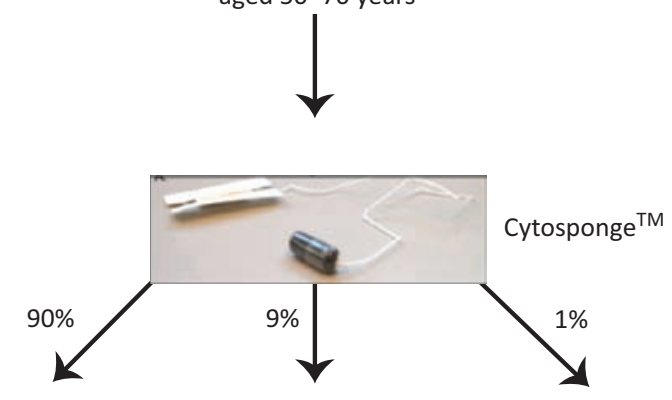

Negative

Low risk positive

High risk positive
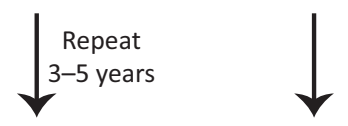

Discharge

Cytosponge $^{\mathrm{TM}}$

Endoscopy and treatment if confirmed

Fig 6. A possible future algorithm for the stratification of patients with Barrett's oesophagus using the Cytosponge ${ }^{\mathrm{TM}}$. 
cells as determined by histology and TFF3, they would undergo further histological and molecular analyses to determine the risk of progression to OAC. On the basis of this stratification, patients might then be offered a further Cytosponge ${ }^{\mathrm{TM}}$ at a later date if they are low risk, or an endoscopy if there is evidence of dysplasia, to treat with the appropriate endoscopic modality.

Although work has started to stratify patients according to clinical, histological and molecular risk, there is more work to be done. Factors, such as a patient's age or sex, body mass index or waist:hip ratio and drug intake, have all been implicated in Barrett's progression and, pending further studies, might have a part in deciding the need for surveillance. Similarly, advanced imaging techniques might enable targeted biopsy in patients who otherwise would undergo standardised sampling. Finally, genetic and molecular markers are likely to have an increasingly important role in predicting those who will progress to OAC and also in diagnosing different degrees of dysplasia.

\section{References}

1 Atkins BZ, Shah AS, Hutcheson KA et al. Reducing hospital morbidity and mortality following esophagectomy. Ann Thorac Surg 2004;78:1170-6.

2 Murray CJL, Richards MA, Newton JN et al. UK health performance: findings of the Global Burden of Disease Study 2010. Lancet 2013;381:997-1020.

3 Ancona E, Rampado S, Cassaro M et al. Prediction of lymph node status in superficial esophageal carcinoma. Ann Surg Oncol 2008; $15: 3278-88$.

4 Rice TW, Zuccaro G, Adelstein DJ et al. Esophageal carcinoma: depth of tumor invasion is predictive of regional lymph node status. Ann Thorac Surg 1998;65:787-92.

5 Jones S, Chen W-D, Parmigiani G et al. Comparative lesion sequencing provides insights into tumor evolution. Proc Natl Acad Sci U S A 2008;105:4283-8.

6 Cairns SR, Scholefield JH, Steele RJ et al. Guidelines for colorectal cancer screening and surveillance in moderate and high risk groups (update from 2002). Gut 2010;59:666-89.

7 Lambert R, Guilloux A, Oshima A et al. Incidence and mortality from stomach cancer in Japan, Slovenia and the USA. Int $J$ Cancer 2002;97:811-8.

8 Hvid-Jensen F, Pedersen L, Drewes AM et al. Incidence of adenocarcinoma among patients with Barrett's esophagus. N Engl J Med 2011;365:375-83.

9 Bhat S, Coleman HG, Yousef F et al. Risk of malignant progression in Barrett's esophagus patients: results from a large populationbased study. J Natl Cancer Inst 2011;103:1049-57.

10 Cooper SC, El-agib A, Dar S et al. Endoscopic surveillance for Barrett's oesophagus: the patients' perspective. Eur J Gastroenterol Hepatol 2009;21:850-4.

11 Rubenstein JH, Sonnenberg A, Davis J et al. Effect of a prior endoscopy on outcomes of esophageal adenocarcinoma among United States veterans. Gastrointest Endosc 2008;68:849-55.

12 Corley DA, Mehtani K, Quesenberry C et al. Impact of endoscopic surveillance on mortality from Barrett's esophagus-associated esophageal adenocarcinomas. Gastroenterology 2013;145:312-9.
13 Fitzgerald RC, Pietro M, Ragunath K et al. British Society of Gastroenterology guidelines on the diagnosis and management of Barrett's oesophagus. Gut 2013;0:1-36.

14 Yousef F, Cardwell C, Cantwell MM, Galway K et al. The incidence of esophageal cancer and high-grade dysplasia in Barrett's esophagus: a systematic review and meta-analysis. Am J Epidemiol 2008;168:237-49.

15 Thomas T, Abrams KR, De Caestecker JS, Robinson RJ. Meta analysis: cancer risk in Barrett's oesophagus. Aliment Pharmacol Ther 2007;26:1465-77.

16 Desai TK, Krishnan K, Samala N et al. The incidence of oesophageal adenocarcinoma in non-dysplastic Barrett's oesophagus: a meta-analysis. Gut 2012;61:970-6.

17 Boerwinkel DF, Swager A-F, Curvers WL, Bergman JJGHM. The clinical consequences of advanced imaging techniques in Barrett's esophagus. Gastroenterology 2014;146:622-9.

18 Smith RR, Hamilton SR, Boitnott JK, Rogers EL. The spectrum of carcinoma arising in Barrett's esophagus. A clinicopathologic study of 26 patients. Am J Surg Pathol 1984;8:563-73.

19 Skinner DB, Walther BC, Riddell RH et al. Barrett's esophagus. Comparison of benign and malignant cases. Ann Surg 1983;198:554-65.

20 Wani S, Falk GW, Post J et al. Risk factors for progression of lowgrade dysplasia in patients with Barrett's esophagus. Gastroenterology 2011;141:1179-86.

21 Curvers WL, ten Kate FJ, Krishnadath KK et al. Low-grade dysplasia in Barrett's esophagus: overdiagnosed and underestimated. Am J Gastroenterol 2010;105:1523-30

22 Phoa KN, van Vilsteren FG, Weusten BL et al. Radiofrequency ablation vs endoscopic surveillance for patients with Barrett's esophagus and low-grade dysplasia: a randomized clinical trial. JAMA 2014;311:1209-17.

23 Skacel M, Petras RE, Rybicki LA et al. p53 expression in low grade dysplasia in Barrett's esophagus: correlation with interobserver agreement and disease progression. Am J Gastroenterol 2002;97:2508-13.

24 Kastelein F, Biermann K, Steyerberg EW et al. Aberrant p53 protein expression is associated with an increased risk of neoplastic progression in patients with Barrett's oesophagus. Gut 2013;62:1676-83.

25 Zagari RM, Fuccio L, Wallander M-A et al. Gastro-oesophageal reflux symptoms, oesophagitis and Barrett's oesophagus in the general population: the Loiano-Monghidoro study. Gut 2008;57:1354-9.

26 Ronkainen J, Aro P, Storskrubb T et al. Prevalence of Barrett's esophagus in the general population: an endoscopic study. Gastroenterology 2005;129:1825-31.

27 Kadri SR, Lao-Sirieix P, O'Donovan M et al. Acceptability and accuracy of a non-endoscopic screening test for Barrett's oesophagus in primary care: cohort study. BMJ 2010;341:c4372.

28 Benaglia T, Sharples LD, Fitzgerald RC, Lyratzopoulos G. Health benefits and cost effectiveness of endoscopic and nonendoscopic cytosponge screening for Barrett's esophagus. Gastroenterology 2013;144:62-73.

Address for correspondence: Prof R Fitzgerald, MRC Cancer Unit, Hutchison-MRC Research Centre, University of Cambridge, Hills Road, Cambridge, CB2 0XZ, UK.

Email: rcf29@mrc-cu.cam.ac.uk 